\title{
Temperature and field independence of the fluorine and lithium NMR shift tensors in
} lithium rare-earth tetrafluorides

\author{
Nevald, Rolf; Hansen, P. E.
}

Published in:

Physical Review B

Link to article, DOI:

10.1103/PhysRevB.18.4626

Publication date:

1978

Document Version

Publisher's PDF, also known as Version of record

Link back to DTU Orbit

Citation (APA):

Nevald, R., \& Hansen, P. E. (1978). Temperature and field independence of the fluorine and lithium NMR shift tensors in lithium rare-earth tetrafluorides. Physical Review B, 18(9), 4626-4634.

https://doi.org/10.1103/PhysRevB.18.4626

\section{General rights}

Copyright and moral rights for the publications made accessible in the public portal are retained by the authors and/or other copyright owners and it is a condition of accessing publications that users recognise and abide by the legal requirements associated with these rights.

- Users may download and print one copy of any publication from the public portal for the purpose of private study or research.

- You may not further distribute the material or use it for any profit-making activity or commercial gain

- You may freely distribute the URL identifying the publication in the public portal 


\title{
Temperature and field independence of the fluorine and lithium NMR shift tensors in lithium rare-earth tetrafluorides
}

\author{
R. Nevald and P. E. Hansen* \\ Department of Electrophysics, The Technical University of Denmark, DK-2800 Lyngby, Denmark
}

(Received 22 June 1978)

\begin{abstract}
The fluorine and lithium NMR line shifts have been followed in temperature from 300 to $1.3 \mathrm{~K}$ and in fields up to $40 \mathrm{kG}$ for $\mathrm{LiTbF}_{4}$ and $\mathrm{LiHoF}_{4}$. The $\mathrm{Tb}^{3+}$ and $\mathrm{Ho}^{3+}$ ionic moments cause these shifts. The $\mathrm{Li}$ shifts are dominated by dipole interactions, whereas the $\mathrm{F}$ shifts also have transferred hyperfine contributions of comparable sizes. The transferred hyperfine interactions turn out to be almost isotropic and exhibiting no temperature or field dependence. In $\mathrm{LiHoF}_{4}$ the line shifts are detectable within the entire temperature range. In $\mathrm{LiTbF}_{4}$ the fluorine and lithium lines broaden to such an extent that they disappear in a region from 10 to $60 \mathrm{~K}$ and from 15 to $40 \mathrm{~K}$, respectively, probably due to a long relaxation time of the Tb ionic moment.
\end{abstract}

\section{INTRODUCTION}

The transferred hyperfine interactions (THI) between the rare-earth ionic moments and the nuclear moments of neighboring diamagnetic ions have been the subject of many investigations, on compounds both dense and dilute in rare-earths. An introduction to the field can be obtained by working backwards through the references of the paper of Reuveni and McGarvey. ${ }^{1}$

We have focused our work on the dense rareearth trifluorides ${ }^{2}$ and lithium rare-earth tetrafluorides. ${ }^{3}$ The tetrafluorides are particularly distinguished by simplicity, which makes them attractive for model studies.

The important qualitative questions about the THI's are whether they are strongly anisotropic or predominantly isotropic, whether they are temperature dependent or not, and whether the isotropic parts are positive or negative. In general there seems to be no unique answer, but for the simple systems the THI's are found to be dominantly isotropic, temperature independent, and negative. This was established in the limited temperature region 77-300 $\mathrm{K}$ for $\mathrm{TbF}_{3}$ and $\mathrm{Dy}_{3}$ in recent work, the limitation being set by NMR line broadening (Hansen et al. ${ }^{2}$ ). Here we report on the results for $\mathrm{LiTbF}_{4}$ and $\mathrm{LiHoF}_{4}$ obtained in the much broader temperature range $1.3-300 \mathrm{~K}$.

\section{THEORY}

The unit cell of the scheelite structure $\left(\mathrm{I} 4_{1} / a\right)$ contains four formula units, but, as outlined in Ref. 3, they all behave equivalently in a magnetic field applied even in a general direction.

The local magnetic field $\overrightarrow{\mathrm{H}}_{n}^{(j)}$ at the $j$ th nucleus of type $n$ ( $\mathrm{Li}$ or $\mathrm{F})$ is

$$
\overrightarrow{\mathrm{H}}_{n}^{(j)}=\overrightarrow{\mathrm{H}}_{a}+\vec{\sigma}_{n}^{(j)} \cdot \overrightarrow{\mathrm{M}} / \rho,
$$

where $\rho$ is the density. For a spherical sample in general, the shift tensor $\vec{\sigma}_{n}^{j}$ consists of three contributions,

$$
\vec{\sigma}_{n}^{(j)}=\zeta_{n} \overrightarrow{1}+\vec{\epsilon}_{n}^{(j)}+\vec{\delta}_{n}^{(j)},
$$

where $\zeta_{n}$ and $\vec{\epsilon}_{n}^{(j)}$ are the isotropic and anisotropic THI's and $\vec{\delta}_{n}^{(j)}$ is the point-dipole contributions given by

$$
\vec{\delta}_{n}^{(j)}=\kappa \sum_{\substack{k \text { in a } \\ \text { sphere }}} \frac{3 \overrightarrow{\mathrm{r}}_{n k}^{(j)} \overrightarrow{\mathrm{r}}_{n k}^{(j)}-\overrightarrow{1}\left|r_{n k}^{(j)}\right|^{2}}{r_{n k}^{(j) 5}},
$$

$\kappa$ being the mass per formula unit and $\overrightarrow{\mathrm{r}}_{n k}^{(j)}$ the vector from the $j$ th ion of type $n$ to the $k$ th $R$ neighbor.

If one of the fluorine shift tensors (assumed symmetric) in the six-notation is written

$$
\vec{\sigma}_{F}^{(1)}=\left(\sigma_{F x x}, \sigma_{F y y}, \sigma_{F z z}, \sigma_{F y z}, \sigma_{F z x}, \sigma_{F x y}\right),
$$

the other three are fixed by symmetry to be

$$
\begin{aligned}
& \vec{\sigma}_{F}^{(2)}=\left(\sigma_{F y y}, \sigma_{F x x}, \sigma_{F z z},-\sigma_{F z x}, \sigma_{F y z},-\sigma_{F x y}\right), \\
& \vec{\sigma}_{F}^{(3)}=\left(\sigma_{F x x}, \sigma_{F y y}, \sigma_{F z z},-\sigma_{F y z},-\sigma_{F z x}, \sigma_{F x y}\right), \\
& \vec{\sigma}_{F}^{(4)}=\left(\sigma_{F y y}, \sigma_{F x x}, \sigma_{F z z}, \sigma_{F z x},-\sigma_{F y z},-\sigma_{F x y}\right) .
\end{aligned}
$$

There is only one lithium shift tensor, and for reasons of symmetry it has the form

$$
\vec{\sigma}_{\mathrm{Li}}=\left(\sigma_{\mathrm{Li}, x x}, \sigma_{\mathrm{Li}, y y}, \sigma_{\mathrm{Li}, z z}, 0,0,0\right) \text {. }
$$

In the experiments presented here the field has always been applied in one of the crystalline axes directions.

This considerably simplifies the following expressions containing only the numerical values of the fields involved, and from the rotational scans presented in Ref. 3 we consider the assignment of the NMR lines as safeguarded.

In the temperature and field ranges where $M$ responds linearly to $\overrightarrow{\mathrm{H}}_{i n}=\overrightarrow{\mathrm{H}}_{a}-\frac{4}{3} \pi M$, one finds for the measurable shift $h \equiv\left(H_{n}^{(j)^{2}}-H_{a}^{2}\right) / 2 H_{a}$, with $\overrightarrow{\mathrm{H}}_{a}$ in the 
$x$ direction (the crystalline $a$ axis)

$$
\begin{aligned}
\frac{h_{\mathrm{F}, a}^{(1,3)}}{\chi_{\perp}(T) H_{a}} \equiv & \frac{\left(H_{\mathrm{F}}^{(1,3)}\right)^{2}-H_{a}^{2}}{2 \chi_{\perp}(T) H_{a}^{2}} \\
= & {\left[N_{\perp}(T)\right]^{-1} \sigma_{\mathrm{F}, x x}+\frac{1}{2} \chi_{\perp}(T)\left[N_{\perp}(T)\right]^{-2} } \\
& \times\left(\sigma_{\mathrm{F}, x x}^{2}+\sigma_{\mathrm{F}, x y}^{2}+\sigma_{\mathrm{F}, z x}^{2}\right) \\
\frac{h_{\mathrm{F}, a}^{(2,4)}}{\chi_{\perp}(T) H_{a}} \equiv & \frac{\left(H_{\mathrm{F}}^{(2,4)}\right)^{2}-H_{a}^{2}}{2 \chi_{\perp}(T) H_{a}^{2}} \\
= & N_{\perp}(T)^{-1} \sigma_{\mathrm{F}, y y}+\frac{1}{2} \chi_{\perp}(T)\left[N_{\perp}(T)\right]^{-2} \\
& \times\left(\sigma_{\mathrm{F}, y y}^{2}+\sigma_{\mathrm{F}, y z}^{2}+\sigma_{\mathrm{F}, x y}^{2}\right),
\end{aligned}
$$

and with $\overrightarrow{\mathrm{H}}_{a}$ in the $z$ direction (the crystalline $c$ axis)

$$
\begin{aligned}
\frac{h_{\mathrm{F}, c}^{(1,2,3,4)}}{\chi_{\|}(T) H_{a}} \equiv & \frac{\left(H_{\mathrm{F}}^{(1,2,3,4)}\right)^{2}-H_{a}^{2}}{2 \chi_{\|}(T) H_{a}^{2}} \\
= & {\left[N_{\|}(T)\right]^{-1} \sigma_{\mathrm{F}, z z}+\frac{1}{2} \chi_{\|}(T)\left[N_{\|}(T)\right]^{-2} } \\
& \quad \times\left(\sigma_{\mathrm{F}, z z}^{2}+\sigma_{\mathrm{F}, z x}^{2}+\sigma_{\mathrm{F}, y z}^{2}\right), \\
\frac{h_{\mathrm{Li}, c}}{\chi_{\|}(T) H_{a}} \equiv & \frac{\left(H_{\mathrm{Li}}\right)^{2}-H_{a}^{2}}{2 \chi_{\|}(T) H_{a}^{2}}=\left[N_{\|}(T)\right]^{-1} \sigma_{\mathrm{Li}, z z},
\end{aligned}
$$

where $N_{\perp}(T)=1+\frac{4}{3} \pi \rho \chi_{\perp}(T)$ and $N_{\|}(T)=1+\frac{4}{3} \pi \rho \chi_{\|}(T)$.

Expressions (7) turn out to be usable in the whole temperature and field range, whereas expressions (8) can only be used for $T \geq 20 \mathrm{~K}$. [It should be noted that the dominant terms in the right-hand side of Eqs. (7) and (8) are those involving the diagonal components of the $\vec{\sigma}_{n}^{(j)}$ only.]

In the case of nonlinear response of $\overrightarrow{\mathrm{M}}$ to $\overrightarrow{\mathrm{H}}$ (i.e., for $T \lessgtr 20 \mathrm{~K}$ and $H$ along the $c$ axis) one gets

$$
\begin{aligned}
\frac{h_{\mathrm{F}, c}^{(1,2,3,4)}}{M_{\|} / \rho} & \equiv \frac{\left(H_{\mathrm{F}}^{(1,2,3,4)}\right)^{2}-H_{a}^{2}}{2 H_{a} M_{\|}\left(T, H_{a}\right) / \rho} \\
& =\sigma_{\mathrm{F}, z z}+\frac{M_{\|}\left(T, H_{a}\right) / \rho}{2 H_{a}} \\
& \times\left(\sigma_{\mathrm{F}, z z}^{2}+\sigma_{\mathrm{F}, z x}^{2}+\sigma_{\mathrm{F}, y z}^{2}\right)
\end{aligned}
$$

and

$$
\frac{h_{\mathrm{Li}, c}}{M_{\|} / \rho} \equiv \frac{\left(H_{\mathrm{Li}}\right)^{2}-H_{a}^{2}}{2 H_{a} M_{\|}\left(T, H_{a}\right) / \rho}=\sigma_{\mathrm{Li}, z z} .
$$

The intrinsic susceptibility components $\chi_{\|}(T)$ and $\chi_{\perp}(T)$ and the magnetization parallel to the $c$ axis $M_{\|}\left(T, H_{a}\right)$ are given in Figs. 1 and 2, respectively, reproduced from the original data of Holmes et $a l .{ }^{4}$ and Hansen et al. ${ }^{5}$

\section{EXPERIMENTAL PROCEDURE}

The single crystals were grown from $99.9 \%$ pure materials by the hydrofluorinating method described by Laursen and Holmes. ${ }^{6}$ The samples were ground into spheres ( 3 and $2 \mathrm{~mm}$ in diameter for $\mathrm{LiTbF}_{4}$ and $\mathrm{LiHoF}_{4}$, respectively), glued to a copper base with Ge 9031, and oriented in situ

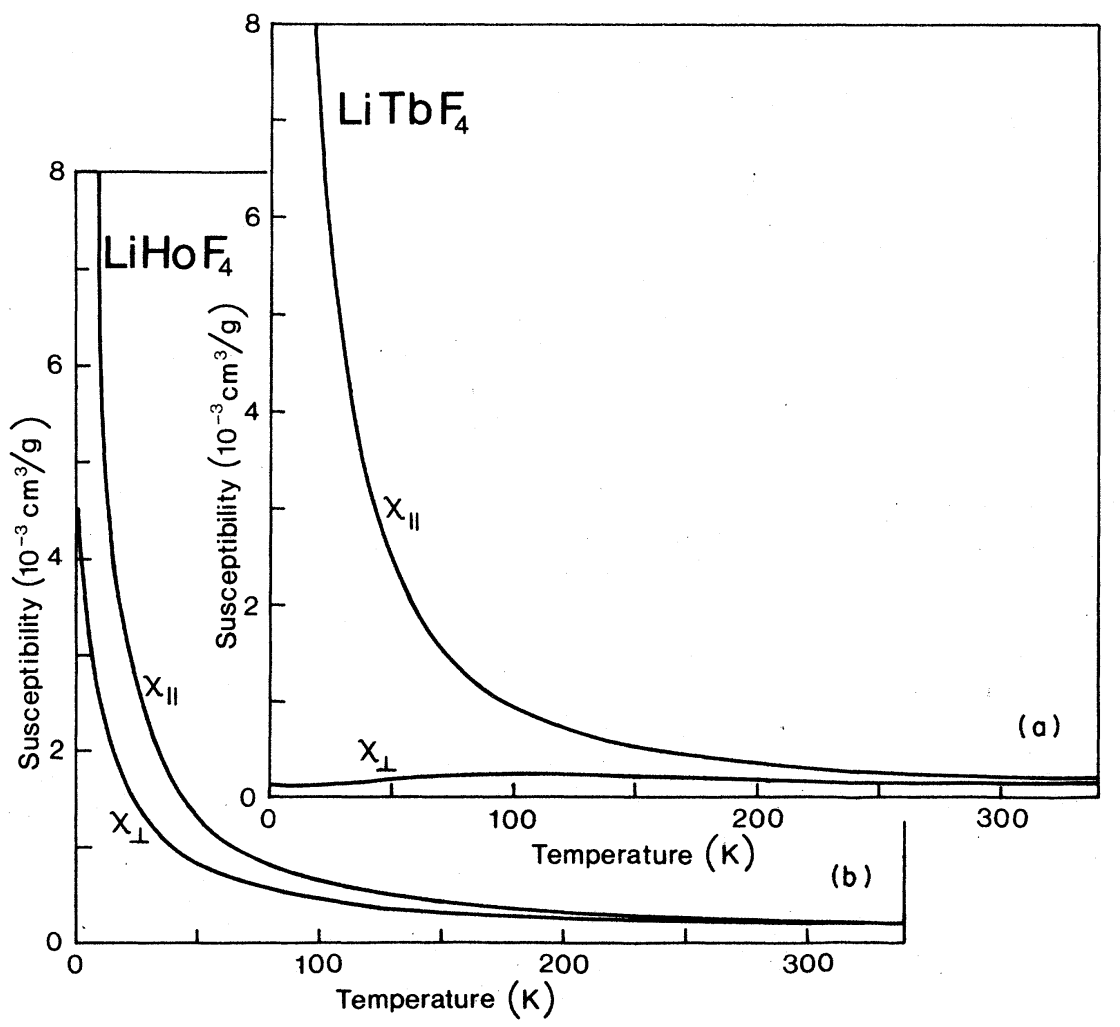

FIG. 1. Intrinsic susceptibility components $\chi_{11}(T)$ and $\chi_{\perp}(T)$. (a) $\mathrm{LiTbF}_{4}$ data from Ref. 4. (b). $\mathrm{LiHoF}_{4}$ data from Ref. 5 . 

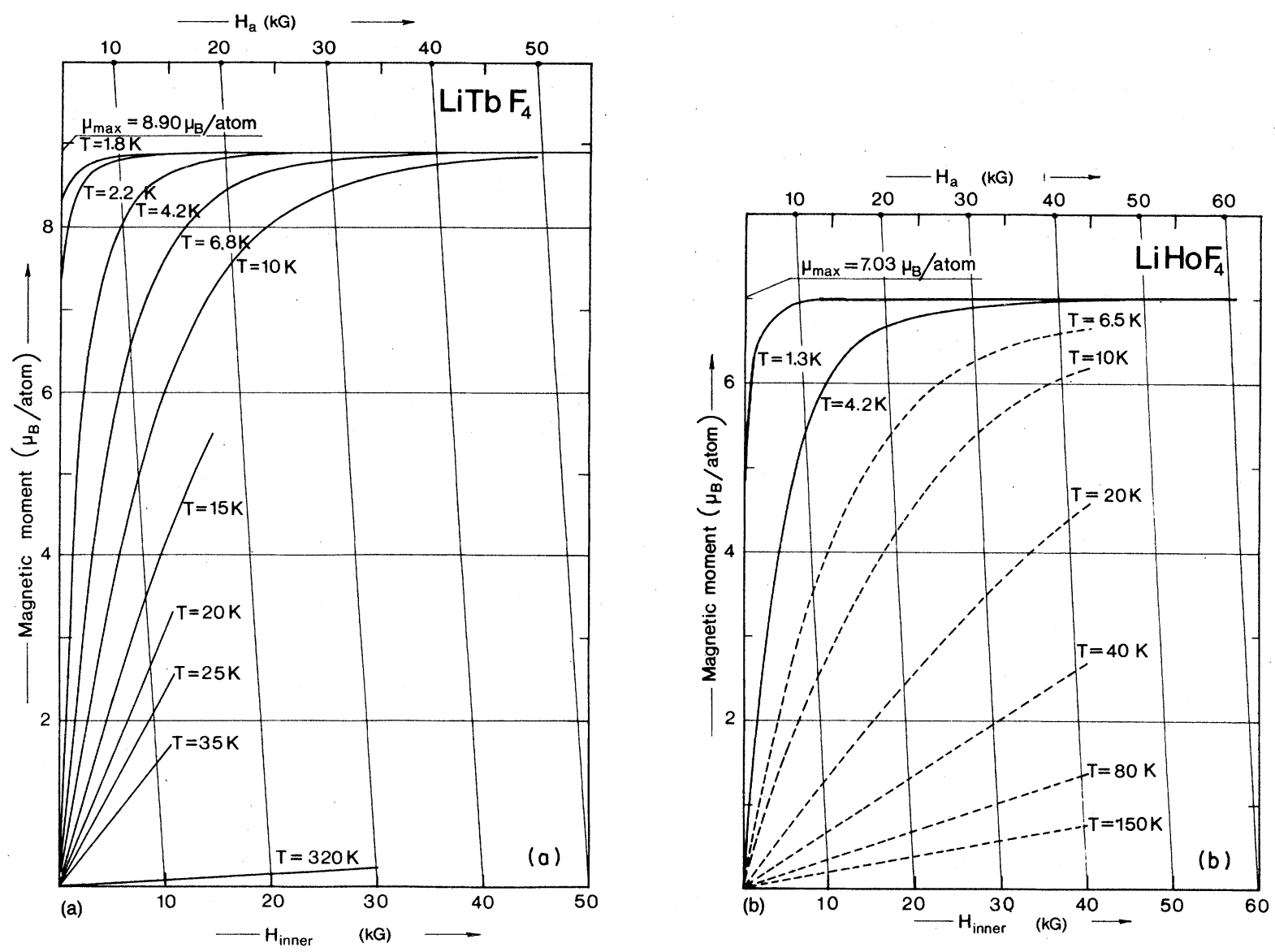

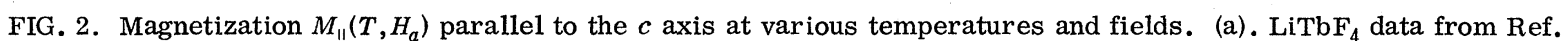
4. (b). Experimental $\mathrm{LiHoF}_{4}$ data from Ref. 5 (solid lines), supplemented with calculated data using crystal field parameters from the same reference (dotted lines).

in the NMR sample holder to better than $1^{\circ}$. A self-supporting five-turns coil was placed around the sample and connected to one of several Robinson-type NMR spectrometers through a $55-\mathrm{cm}$ coaxial line. For the high field ${ }^{19} \mathrm{~F}$ spectra a special high-frequency NMR spectrometer capable of working up to $300 \mathrm{MHz}$ was used. ${ }^{7}$

All spectra were obtained using field modulation and phase-sensitive detection. The spectrometer frequency was kept fixed and the magnetic field swept through the resonances. The measurements below $77 \mathrm{~K}$, called in the following the lowtemperature measurements, were performed in a superconducting magnet, whose field was monitored with an accuracy of \pm 3 Oe through a precision measurement of the magnet current. (The magnet wire is of the twisted multifilament type, for which current loops are precluded.) The hightemperature measurements, those above $77 \mathrm{~K}$, were taken in an electromagnet, and the field was determined with an NMR gaussmeter, giving an accuracy of $\pm 50 \mathrm{ppm}$.

The sample temperatures was obtained from the resistance of an Allen-Bradley carbon resistor below $40 \mathrm{~K}$ and of a $\mathrm{Cu}$ wire above $40 \mathrm{~K}$. The temperature regulator kept the temperature fixed to better than $\pm 0.5 \%$ during a spectral scan. For further details of the setup, Nevald and Hansen ${ }^{8}$ may be consulted.

The high-temperature measurements were taken for the magnetic field $H_{a}$ along the $c$ as well as the $a$ axes. The low-temperature measurements were taken with $H_{a}$ along the $c$ axis only, because the torque shattered the crystals when $H_{a}$ was sufficiently strong for NMR measurements and applied along the $a$ axis.

All NMR lines broaden on going down in temperature from $300 \mathrm{~K}$, but whereas for $\mathrm{LiHoF}_{4}$ they stay observable in the whole temperature range, for $\mathrm{LiTbF}_{4}$ the $\mathrm{F}$ lines disappear around $60 \mathrm{~K}$ and the $\mathrm{Li}$ lines at $40 \mathrm{~K}$, probably due to decreasing relaxation rate of the Tb ionic mo- 
ment. The $\mathrm{F}$ and $\mathrm{Li}$ lines reappear for the highest applied fields at 10 and $15 \mathrm{~K}$, respectively, due to the increasing order in the $\mathrm{Tb}$ ionic moments. These observations will be treated in a separate paper.

\section{RESULTS}

The ${ }^{7} \mathrm{Li}$ line shifts in $\mathrm{LiTbF}_{4}$ and $\mathrm{LiHoF}_{4}$, $h_{\mathrm{Li}}\left(T, H_{a}\right)=\left(H_{\mathrm{Li}}^{2}-H_{a}^{2}\right) / 2 H_{a}$ measured at various temperatures and fields are shown in Table I. The corresponding magnetizations or susceptibilities, needed for the determination of $\sigma_{z z}$, are also included and, finally, the resulting values of $\sigma_{z z}$ are quoted. The mean values of $\sigma_{z z}$ and their standard deviations are extracted and collected in Table IV.

Similarly the ${ }^{19} \mathrm{~F}$ line shifts in $\mathrm{LiTbF}_{4}$ and $\mathrm{LiHoF}_{4}, h_{\mathrm{F}}^{(j)}\left(T, H_{a}\right)=\left(H_{\mathrm{F}}^{(j) 2}-H_{a}^{2}\right) / 2 H_{a}$ are given in Tables II and III, respectively. By the method of least squares, the components of the shift tensors, taken to be temperature and field independent, are determined using Eqs. (7)-(9), and their standard deviations are calculated. (The diagonal and off-diagonal shift components are collected in Tables IV and VI, respectively.) From the fitted shift tensors the two contributions to the measured shifts are calculated and included in Tables

TABLE I. Measured ${ }^{7} \mathrm{Li}$ shifts for various temperatures and fields along the $c$ direction, and the corresponding values of $\sigma_{z z}$. For convenience, the magnetization $M_{\|}$or susceptibility $\chi_{\|}$(as relevant) is also included.

\begin{tabular}{|c|c|c|c|c|}
\hline $\begin{array}{l}\text { Temp. } \\
\text { (K) }\end{array}$ & $\begin{array}{c}M_{\|} / \rho \\
\left(\mathrm{Oe} \mathrm{cm}^{3} / \mathrm{g}\right) \\
\text { or } \\
\chi_{\|} \\
\left(10^{3} \mathrm{~cm}^{3} / \mathrm{g}\right)\end{array}$ & $\begin{array}{c}H_{a} \\
\text { (Oe) }\end{array}$ & $\begin{array}{c}\begin{array}{c}\text { Shift } \\
H_{\mathrm{Li}}^{2}-H_{a}^{2}\end{array} \\
\frac{2 H_{a}}{(\mathrm{Oe})}\end{array}$ & $\begin{array}{c}\sigma_{z z} \\
\left(\mathrm{~g} / \mathrm{cm}^{3}\right)\end{array}$ \\
\hline \multicolumn{5}{|c|}{${ }^{7} \mathrm{Li}$ res. in $\mathrm{LiTbF}_{4}$} \\
\hline 1.3 & 206 & 37741 & -1844 & -8.95 \\
\hline 4.2 & 205 & 25932 & -1804 & -8.80 \\
\hline 4.2 & 206 & 33754 & -1822 & -8.84 \\
\hline 4.2 & 206 & 37896 & -1825 & -8.86 \\
\hline 4.9 & 206 & 37779 & -1824 & -8.86 \\
\hline 5.2 & 206 & 33653 & -1825 & -8.86 \\
\hline 5.7 & 206 & 37777 & -1826 & -8.87 \\
\hline 6.0 & 205 & 33648 & -1820 & -8.88 \\
\hline 7.6 & 204 & 37797 & -1823 & -8.85 \\
\hline 8.0 & 202 & 33608 & -1821 & -9.00 \\
\hline 8.8 & 202 & 33650 & -1837 & -9.00 \\
\hline 10.0 & 199 & 33638 & -1810 & -9.04 \\
\hline 54 & 2.222 & 32380 & -613 & -9.00 \\
\hline 61 & 2.195 & 32289 & -525 & -9.03 \\
\hline 75 & 1.416 & 32142 & -405 & -9.00 \\
\hline 86 & 1.175 & 32082 & -326 & -8.90 \\
\hline 96 & 0.987 & 32028 & -269 & -8.70 \\
\hline 105 & 0.868 & 31988 & -235 & -8.64 \\
\hline 137 & 0.593 & 31925 & -160 & -8.55 \\
\hline 162 & 0.470 & 31891 & -125 & -8.65 \\
\hline 301 & 0.208 & 8310 & -16.0 & -9.23 \\
\hline \multicolumn{5}{|c|}{${ }^{7} \mathrm{Li}$ res. in $\mathrm{LiHoF}_{4}$} \\
\hline 4.2 & 153 & 26153 & -1515 & -9.9 \\
\hline 4.2 & 156 & 31004 & -1513 & -9.7 \\
\hline 4.2 & 157 & 36325 & -1633 & -10.4 \\
\hline 12 & 127 & 36100 & -1422 & -11.2 \\
\hline 51 & 1.316 & 35875 & -434 & -9.2 \\
\hline 103 & 0.641 & 35670 & -219 & -9.6 \\
\hline 163 & 0.402 & 35594 & -145 & -10.1 \\
\hline 302 & 0.204 & 8527 & -17.4 & -10.0 \\
\hline
\end{tabular}


TABLE II. Measured ${ }^{19} \mathrm{~F}$ line shifts in $\mathrm{LiTbF}_{4}, h_{F}\left(T, H_{a}\right)=\left(H_{F}^{2}-H_{a}^{2}\right) / 2 H_{a}$ for various temperatures and fields, applied along the crystallographic directions. The shifts are further divided into contribution due to the two parts of the shift tensors, $\sigma_{\alpha \alpha}$ and $\sigma_{\alpha \beta}^{2}+\sigma_{\gamma \alpha}^{2}+\sigma_{\alpha \alpha}^{2}$. Also the difference $\Delta$ between the measured shifts and the calculated contributions is included.

\begin{tabular}{|c|c|c|c|c|c|c|c|}
\hline & $\begin{array}{l}\text { Temp. } \\
\text { (K) }\end{array}$ & $\begin{array}{c}M_{\|} / \rho \\
\left(\mathrm{Oe} \mathrm{cm}^{3} / \mathrm{g}\right) \\
\text { or } \\
\chi_{\|} \\
\left(10^{3} \mathrm{~cm}^{3} / \mathrm{g}\right)\end{array}$ & $\begin{array}{c}H_{a} \\
(\mathrm{Oe})\end{array}$ & $\begin{array}{c}\begin{array}{c}\text { Shift } \\
H_{F}^{2}-H_{a}^{2}\end{array} \\
2 H_{a} \\
(\mathrm{Oe})\end{array}$ & $\begin{array}{l}\text { Shift } \\
\text { due to } \\
\sigma_{\alpha \alpha} \\
(\mathrm{Oe})\end{array}$ & $\begin{array}{c}\text { Shift } \\
\text { due to } \\
\sigma_{\alpha \beta}^{2}+\sigma_{\gamma \alpha}^{2}+\sigma_{\alpha \alpha}^{2} \\
\text { (Oe) }\end{array}$ & $\begin{array}{c}\Delta \\
(\mathrm{Oe})\end{array}$ \\
\hline$\vec{H}_{a} \| \vec{z}$ & 1.3 & 206 & 31720 & -907 & -2724 & 1863 & 46 \\
\hline$F^{(1,2,3,4)}$ & 4.2 & 203 & 12083 & 2105 & -2684 & 4752 & -37 \\
\hline \multirow{39}{*}{$(\alpha=z)$} & 4.2 & 203 & 13304 & 1670 & -2684 & 4315 & -39 \\
\hline & 4.2 & 204 & 16007 & 921 & -2698 & 3622 & 3 \\
\hline & 4.2 & 204 & 16767 & 771 & -2698 & 3460 & -9 \\
\hline & 4.2 & 205 & 22367 & -123 & -2711 & 2619 & 30 \\
\hline & 4.2 & 205 & 27245 & -586 & -2711 & 2149 & 24 \\
\hline & 4.2 & 205 & 29785 & -763 & -2711 & 1966 & 18 \\
\hline & 4.2 & 205 & 37118 & -1160 & -2711 & 1578 & 26 \\
\hline & 4.2 & 205 & 39297 & -1227 & -2711 & 1490 & 6 \\
\hline & 5.2 & 205 & 27827 & -615 & -2711 & 2104 & 7 \\
\hline & 5.5 & 205 & 27798 & -640 & -2711 & 2106 & 35 \\
\hline & 5.6 & 205 & 27851 & -634 & -2711 & 2102 & 25 \\
\hline & 6.0 & 205 & 27827 & -622 & -2711 & 2108 & 18 \\
\hline & 6.0 & 205 & 27798 & -639 & -2711 & 2101 & 29 \\
\hline & 6.0 & 205 & 27799 & -633 & -2711 & 2106 & 28 \\
\hline & 6.5 & 204 & 27786 & -622 & -2708 & 2086 & 0 \\
\hline & 6.5 & 204 & 27755 & -610 & -2698 & 2089 & 1 \\
\hline & 6.5 & 204 & 27840 & -631 & -2699 & 2082 & 14 \\
\hline & 7.0 & 203 & 27770 & -609 & -2686 & 2068 & -9 \\
\hline & 7.0 & 203 & 277.49 & -606 & -2686 & 2070 & -10 \\
\hline & 7.0 & 203 & 27836 & -625 & -2686 & 2063 & 2 \\
\hline & 7.3 & 203 & 27764 & -605 & -2686 & 2069 & -12 \\
\hline & 7.6 & 202 & 27750 & -605 & -2672 & 2050 & -17 \\
\hline & 7.6 & 202 & 27840 & -631 & -2673 & 2042 & 1 \\
\hline & 7.9 & 202 & 37509 & -1177 & -2672 & 1514 & 19 \\
\hline & 8.0 & 202 & 27780 & -621 & -2673 & 2047 & -5 \\
\hline & 8.5 & 201 & 27820 & -612 & -2659 & 2024 & -23 \\
\hline & 9.0 & 200 & 27806 & -596 & -2646 & 2005 & -44 \\
\hline & 60.0 & 2.024 & 36994 & -712 & -948 & 211 & -24 \\
\hline & 72.0 & 1.501 & 36837 & -615 & -707 & 116 & -23 \\
\hline & 89.0 & 1.103 & 36669 & -453 & -522 & 62 & -7 \\
\hline & 101 & 0.919 & 8157 & -93.3 & -99.3 & 9.6 & 3.6 \\
\hline & 113 & 0.781 & 36554 & -330 & -369 & 31 & -7 \\
\hline & 128 & 0.658 & 32590 & -255 & -280 & 20 & -5 \\
\hline & 142 & 0.562 & 32559 & -212 & -239 & 14 & -13 \\
\hline & 161 & 0.476 & 32541 & -183 & -203 & 10 & -10 \\
\hline & 171 & 0.436 & 8149 & -45.5 & -46.4 & 2.2 & 1.2 \\
\hline & 201 & 0.351 & 8147 & -36.8 & -37.5 & 1.4 & 0.7 \\
\hline & 308 & 0.202 & 8042 & -20.8 & -21.5 & 0.5 & -0.1 \\
\hline & & & & & & & \\
\hline$\vec{H}_{a} \| \vec{x}$ & 80 & 0.235 & 8069.2 & -13.8 & -14.6 & 1.0 & 0.2 \\
\hline$F^{(2,4)}$ & 97 & 0.241 & 8074.5 & -13.7 & -15.0 & 1.1 & -0.2 \\
\hline$(\alpha=y)$ & 126 & 0.230 & 8081.8 & -13.0 & -14.3 & 1.1 & -0.2 \\
\hline & 163 & 0.206 & 8060.8 & -12.0 & -12.8 & 0.8 & 0 \\
\hline & 205 & 0.180 & 8052.9 & -11.2 & -11.2 & 0.6 & 0.6 \\
\hline & 253 & 0.155 & 8040.5 & -8.9 & -9.6 & 0.5 & -0.3 \\
\hline & 304 & 0.135 & 8037.3 & -8.0 & -8.4 & 0.4 & 0 \\
\hline
\end{tabular}


TABLE II. (Continued)

\begin{tabular}{|c|c|c|c|c|c|c|c|}
\hline & $\begin{array}{l}\text { Temp. } \\
\text { (K) }\end{array}$ & 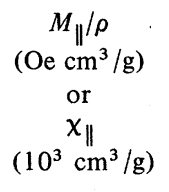 & $\begin{array}{c}H_{a} \\
(\mathrm{Oe})\end{array}$ & $\begin{array}{c}\text { Shift } \\
\frac{H_{F}^{2}-H_{a}^{2}}{2 H_{a}} \\
(\mathrm{Oe})\end{array}$ & $\begin{array}{l}\text { Shift } \\
\text { due to } \\
\sigma_{\alpha \alpha} \\
(\mathrm{Oe})\end{array}$ & $\begin{array}{c}\text { Shift } \\
\text { due to } \\
\sigma_{\alpha \beta}^{2}+\sigma_{\gamma \alpha}^{2}+\sigma_{\alpha \alpha}^{2} \\
\text { (Oe) }\end{array}$ & $\begin{array}{c}\Delta \\
\text { (Oe) }\end{array}$ \\
\hline$\vec{H}_{a} \| \vec{x}$ & 80 & 0.235 & 8121.3 & -66.5 & -67.9 & 1.0 & -0.4 \\
\hline$F^{(1,3)}$ & 97 & 0.241 & 8128.9 & -68.2 & -69.7 & 1.0 & -0.5 \\
\hline \multirow{5}{*}{$(\alpha=x)$} & 126 & 0.230 & 8135.3 & -65.9 & -66.6 & 1.0 & 0.3 \\
\hline & 163 & 0.206 & 8106.4 & -59.2 & -59.5 & 0.8 & 0.9 \\
\hline & 205 & 0.180 & 8094.1 & -52.2 & -52.0 & 0.6 & 0.8 \\
\hline & 253 & 0.155 & 8074.5 & -44.1 & -44.6 & 0.4 & -0.1 \\
\hline & 304 & 0.135 & 8068.2 & -38.6 & -38.9 & 0.3 & 0 \\
\hline
\end{tabular}

TABLE III. Measured ${ }^{19} \mathrm{~F}$ shifts in $\mathrm{LiHoF}_{4}, h_{F}\left(T, H_{a}\right)=\left(H_{F}^{2}-H_{a}^{2}\right) / 2 H_{a}$, for various temperatures and fields applied along the crystallographic axes. The shifts are divided into contributions due to the two parts of the shift tensors, $\sigma_{\alpha \alpha}$ and $\sigma_{\alpha \beta}^{2}+\sigma_{\gamma \alpha}^{2}+\sigma_{\alpha \alpha}^{2}$. Also the difference $\Delta$ between the measured shifts and the calculated contributions is included.

\begin{tabular}{|c|c|c|c|c|c|c|c|}
\hline & $\begin{array}{c}\text { Temp. } \\
(\mathrm{K})\end{array}$ & $\begin{array}{c}M_{\|} / \rho \\
\left(\mathrm{Oe} \mathrm{cm}^{3} / \mathrm{g}\right) \\
\text { or } \\
\chi_{\|} \\
\left(10^{3} \mathrm{~cm}^{3} / \mathrm{g}\right)\end{array}$ & $\begin{array}{c}H_{a} \\
(\mathrm{Oe})\end{array}$ & $\begin{array}{c}\begin{array}{c}\text { Shift } \\
H_{F}^{2}-H_{a}^{2}\end{array} \\
2 H_{a} \\
(\mathrm{Oe})\end{array}$ & $\begin{array}{l}\text { Shift } \\
\text { due to } \\
\sigma_{\alpha \alpha} \\
(\mathrm{Oe})\end{array}$ & $\begin{array}{c}\text { Shift } \\
\text { due to } \\
\sigma_{\alpha \beta}^{2}+\sigma_{\gamma \alpha}^{2}+\sigma_{\alpha \alpha}^{2} \\
\text { (Oe) }\end{array}$ & $\begin{array}{c}\Delta \\
(\mathrm{Oe})\end{array}$ \\
\hline$\vec{H}_{a} \| \vec{z}$ & 4.2 & 152 & 22111 & 1457 & -902 & 2401 & 42 \\
\hline$F^{(1,2,3,4)}$ & 4.2 & 155 & 27690 & 1093 & -919 & 1994 & -18 \\
\hline \multirow{11}{*}{$\alpha=z$} & 4.2 & 157 & 35642 & 702 & -931 & 1589 & -44 \\
\hline & 38 & 1.786 & 36559 & -116 & -371 & 247 & -8 \\
\hline & 52 & 1.290 & 36571 & -127 & -271 & 132 & -12 \\
\hline & 65 & 1.020 & 36594 & -147 & -216 & 83 & 14 \\
\hline & 85 & 0.770 & 8339.5 & -27.7 & -37.4 & 11.0 & 1.2 \\
\hline & 110 & 0.592 & 8297.1 & -22.7 & -28.7 & 6.5 & 0.5 \\
\hline & 140 & 0.462 & 8289.5 & -18.1 & -22.5 & 4.0 & -0.3 \\
\hline & 160 & 0.402 & 8271.0 & -16.6 & -19.5 & 3.0 & 0.0 \\
\hline & 185 & 0.344 & 8261.5 & -14.7 & -16.7 & 2.2 & 0.2 \\
\hline & 210 & 0.302 & 8266.4 & -12.8 & -14.7 & 1.7 & -0.2 \\
\hline & 240 & 0.262 & 8298.3 & -11.4 & -12.8 & 1.3 & -0.1 \\
\hline$\vec{H}_{a} \| \vec{x}$ & 85 & 0.534 & 8304.8 & 7.5 & 3.6 & 4.3 & 0.4 \\
\hline$F^{(2,4)}$ & 110 & 0.426 & 8224.4 & 6.4 & 2.9 & 2.7 & -0.8 \\
\hline \multirow[t]{5}{*}{$\alpha=y$} & 140 & 0.344 & 8266.1 & 3.6 & 2.3 & 1.8 & 0.5 \\
\hline & 160 & 0.304 & 8283.2 & 3.1 & 2.1 & 1.4 & 0.4 \\
\hline & 185 & 0.268 & 8256.2 & 3.5 & 1.8 & 1.1 & -0.6 \\
\hline & 210 & 0.240 & 8247.7 & 2.4 & 1.6 & 0.9 & 0.1 \\
\hline & 240 & 0.212 & 8321.3 & 2.1 & 1.5 & 0.7 & 0.0 \\
\hline$\vec{H}_{a} \| \vec{x}$ & 85 & 0.534 & 8436.6 & -142.1 & -143.4 & 0.6 & -0.8 \\
\hline$F^{(1,3)}$ & 110 & 0.426 & 8327.1 & -113.1 & -113.3 & 0.4 & 0.2 \\
\hline \multirow{5}{*}{$\alpha=x$} & 140 & 0.344 & 8381.0 & -93.1 & -92.3 & 0.2 & 1.1 \\
\hline & 160 & 0.304 & 8348.6 & -82.2 & -81.3 & 0.2 & 1.0 \\
\hline & 185 & 0.268 & 8332.2 & -71.8 & -71.6 & 0.1 & 0.3 \\
\hline & 210 & 0.240 & 8310.0 & -63.5 & -64.0 & 0.1 & -0.4 \\
\hline & 240 & 0.212 & 8386.2 & -56.3 & -57.1 & 0.1 & -0.7 \\
\hline
\end{tabular}


TABLE IV. Shift tensor diagonal components and the isotropic THI for ${ }^{7} \mathrm{Li}$ and ${ }^{19} \mathrm{~F}$ in $\mathrm{LiTbF}_{4}$ and $\mathrm{LiHoF}_{4}$ in $\mathrm{g} / \mathrm{cm}^{3}$.

\begin{tabular}{lccccc}
\hline & & & & $\mathrm{LiHoF}_{4}$ & \\
& ${ }^{19} \mathrm{~F}$ & $\mathrm{LiTbF}_{4}$ & ${ }^{7} \mathrm{Li}$ & ${ }^{19} \mathrm{~F}$ & ${ }^{7}$ \\
\hline$\sigma_{x x}$ & $-35.81 \pm 0.82$ & $4.4 \pm 1^{\mathrm{a}}$ & $-32.26 \pm 0.40$ & $5.0 \pm 1^{\mathrm{a}}$ \\
$\sigma_{y y}$ & $-7.75 \pm 0.46$ & $4.4 \pm 1^{\mathrm{a}}$ & $0.81 \pm 0.24$ & $5.0 \pm 1^{\mathrm{a}}$ \\
$\sigma_{z z}$ & $-13.20 \pm 0.12$ & $-8.88 \pm 0.18$ & $-6.00 \pm 0.09$ & $-10.0 \pm 0.6$ \\
$\zeta$ & $-18.92 \pm 0.94$ & $0 \quad \pm 1$ & $-12.48 \pm 0.48$ & $0 \quad \pm 1$ \\
\hline \hline
\end{tabular}

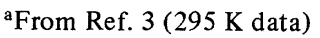

II and III. Finally, the difference between the sum of the two calculated shift contributions and the measured shifts are given.

To give a further impression of the experimental material, the shifts are plotted for $\mathrm{LiTbF}_{4}$ with $H_{a}$ applied along the $c$ axis in Fig. 3. To the left the shifts are shown at $4.2 \mathrm{~K}$ for varied field, and to the right the shifts are given at $33 \mathrm{kG}$ for varied temperature. For higher temperatures, where the shifts get relatively small, they are also drawn in a fourfold expanded scale. The top figure presents the ${ }^{7} \mathrm{Li}$ shifts, whereas the bottom figure shows the ${ }^{19} \mathrm{~F}$ shifts. For ${ }^{19} \mathrm{~F}$ the two contributions from different parts of the shift tensors are also plotted. It can be seen that although the shifts and the relative magnitudes of the two contributions vary strongly, the fitting can be done without resorting to temperature- or field-dependent shift tensors.

We have chosen to present the experimental material rather extensively to demonstra te that at least for these crystals the temperature and field dependence of the shift tensors are indeed negligible.

\section{DISCUSSION}

In our earlier paper on NMR in lithium rareearth tetrafluorides ${ }^{3}$ all the components of the shift tensors at room temperature were measured. Thereby it was found that the isotropic THI with the $\mathrm{F}$ nuclei was negative and comparable in size to the dipole tensor components, whereas the anisotropic THI was less than $10 \%$ of the dipole tensor and probably down in the experimental noise.

In this paper we have chosen to follow closely some selected tensor components through a large temperature region and up to $40 \mathrm{kG}$ in applied field. These are primarily the diagonal components $\sigma_{x x}, \sigma_{y y}$, and $\sigma_{z z}$ and, to a lesser degree, $\sigma_{z x}^{2}+\sigma_{y z}^{2}$, which appear in Eqs. (8) and (9). [ $\sigma_{x y}^{2}$ $+\sigma_{z x}^{2}$ and $\sigma_{y z}^{2}+\sigma_{x y}^{2}$ enter Eqs. (7) with so little weight that the experiments render them very inaccurate]. It has to be mentioned that although the shifts increase up to 100 times on cooling to low temperature, the linewidth increases, too, from $\sim 6 \mathrm{G}$ at room temperature through very large values around $30 \mathrm{~K}$ to $\sim 50 \mathrm{G}$ at liquid-He temperatures. This means that the accuracy of the determination of the shift tensor components rises at most a factor of 10 on expanding the temperature region. Still, this means an improvement on the accuracy of $\sigma_{x x}, \sigma_{y y}$, and $\sigma_{z z}$.

The most important fact, however, learned from the present measurements - as can be seen from Tables I-III-is that inside the experimental accuracy, $\sigma_{x x}, \sigma_{y y}, \sigma_{z z}$, and $\sigma_{z x}^{2}+\sigma_{y z}^{2}$ are temperature independent between 1.3 and $300 \mathrm{~K}$, and field independent up to $40 \mathrm{kG}$.

Also the soundness of the basic expression (1) for the local field seems rather convincingly demonstrated, considering the very different conditions at $300 \mathrm{~K}$, where the magnetic ions are slightly polarized only, and at $4.2 \mathrm{~K}$, where they are nearly saturated. Furthermore, at room tem-

TABLE V. Diagonal components of the anisotropic THI tensor for ${ }^{7} \mathrm{Li}$ and ${ }^{19} \mathrm{~F}$ in $\mathrm{LiTbF}_{4}$ and $\mathrm{LiHoF}_{4}$ in $\mathrm{g} / \mathrm{cm}^{3}$. All components are zero inside the experimental accuracy.

\begin{tabular}{|c|c|c|c|c|}
\hline & \multicolumn{2}{|c|}{$\mathrm{LiTbF}_{4}$} & \multicolumn{2}{|c|}{$\mathrm{LiHoF}_{4}$} \\
\hline & ${ }^{19} \mathrm{~F}$ & ${ }^{7} \mathrm{Li}$ & ${ }^{19} \mathrm{~F}$ & ${ }^{7} \mathrm{Li}$ \\
\hline$\epsilon_{x x}$ & $1.2 \pm 2.2$ & $-0.4 \pm 1$ & $-0.7 \pm 2.0$ & $0.0 \pm 1$ \\
\hline$\epsilon_{y y}$ & $-0.8 \pm 2.0$ & $-0.4 \pm 1$ & $0.6 \pm 2.0$ & $0.0 \pm 1$ \\
\hline$\epsilon_{z z}$ & $-0.4 \pm 2.0$ & $0.8 \pm 1$ & $0.1 \pm 2.0$ & $0.0 \pm 1$ \\
\hline
\end{tabular}



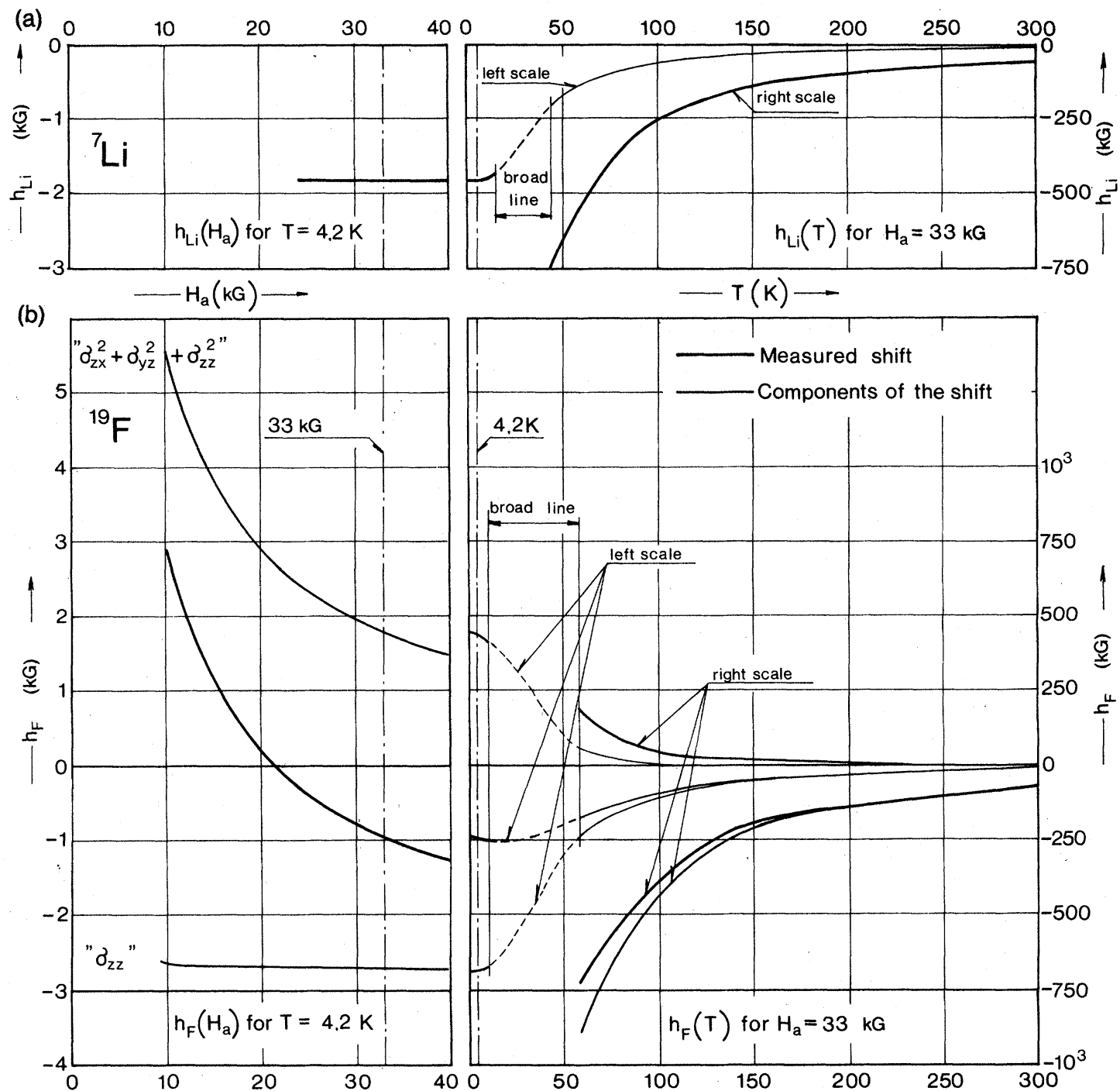

FIG. 3. Measured line shifts in $\mathrm{LiTbF}_{4}$ with $\boldsymbol{H}_{a}$ along the $c$ axis for various fields and temperatures. (a). ${ }^{7} \mathrm{Li}$ line shifts. (b). ${ }^{19} \mathrm{~F}$ line shifts. Their divisions in shifts due to $\sigma_{F, z z}$ and $\left(\sigma_{F, z z}^{2}+\sigma_{F, z x}^{2}+\sigma_{F, y z}^{2}\right)$ are also shown.

TABLE VI. Shift tensor off-diagonal components for ${ }^{19} \mathrm{~F}$ in $\mathrm{LiTbF}_{4}$ and $\mathrm{LiHoF}_{4}$ compared to those calculated from Ref. 3 and from the dipole tensor. (All values in $\mathrm{g} / \mathrm{cm}^{3}$.) The uncertainty of $\pm 3 \mathrm{~g} / \mathrm{cm}^{3}$ on the dipole tensor combinations is due to lack of knowledge of the exact fluorine positions.

\begin{tabular}{|c|c|c|c|c|c|c|}
\hline \multirow{2}{*}{$\begin{array}{l}{ }^{19} \mathrm{~F} \\
\alpha \beta \gamma\end{array}$} & \multicolumn{3}{|c|}{$\mathrm{LiTbF}_{4}$} & \multicolumn{3}{|c|}{$\mathrm{LiHoF}_{4}$} \\
\hline & This work & Ref. 3 & $\left(\delta_{\alpha \beta}^{2}+\delta_{\gamma \alpha}^{2}\right)^{1 / 2}$ & This work & Ref. 3 & $\left(\delta_{\alpha \beta}^{2}+\delta_{\gamma \alpha}^{2}\right)^{1 / 2}$ \\
\hline$x y z$ & $\pm 40)$ & $23 \pm 1$ & $23 \pm 3$ & $(0 \pm 50)$ & $24 \pm 1$ & $25 \pm 3$ \\
\hline$y z x$ & $(60 \pm 50)$ & $53 \pm 1$ & $57 \pm 3$ & $(61 \pm 12)$ & $61 \pm 1$ & $60 \pm 3$ \\
\hline$z x y$ & $52.0 \pm 0.5$ & $52 \pm 1$ & $56 \pm 3$ & $66 \pm 1$ & $63 \pm 1$ & $60 \pm 3$ \\
\hline
\end{tabular}


perature all levels of the magnetic ion are significantly populated, whereas at liquid-He temperatures only the lowest level contributes to the magnetization.

From $\sigma_{n x x}, \sigma_{n y y}$, and $\sigma_{n z z}$, the isotropic THI's $\zeta_{n}$ $=\frac{1}{3}\left(\sigma_{n x x}+\sigma_{n y y}+\sigma_{n z z}\right)$ can be determined, and they are included in Table IV.

$\zeta_{\mathrm{F}}$ is negative for both $\mathrm{LiTbF}_{4}$ and $\mathrm{LiHoF}_{4}$, and the absolute value is smallest for $\mathrm{LiHoF}_{4}$, which has the smallest $f$-shell radius. (The present value of $\zeta_{\mathrm{F}}$ for $\mathrm{LiHoF}_{4}$ is different from the earlier reported value from the $295-\mathrm{K}$ data ${ }^{3}$ considering the claimed accuracy. As the larger amount of experimental data in the present work has allowed us to calculate the accuracy, we believe the estimated accuracy in the earlier work to be too optimistic.)

$\zeta_{\mathrm{Li}}$ is found to be zero for both materials. This was to be expected, the $\mathrm{Li}$ ions being electropositive and far away from the magnetic ions.

In this paper we are primarily focusing on the diagonal components of the shift tensors. In order to determine whether there are nonzero anisotropic THI diagonal components, the isotropic THI and the dipole tensor diagonal components (calculated in Ref. 3) are subtracted from the corresponding components of the measured shift tensors. The results for both $\mathrm{Li}$ and $\mathrm{F}$ sites are presented in Table V. It is seen that all anisotropic THI components are zero inside the experimental accuracy.

The off-diagonal components of the shift tensors are only of secondary interest in this work. Still, the measured combinations as well as those found at $295 \mathrm{~K}$ (Ref. 3) are compared (Table VI) to the corresponding combinations of the dipole tensor components. Only the $z x$ and $y z$ combinations are well determined in this study. Their values, which we have shown to hold in the whole temperature region $1.3-300 \mathrm{~K}$, are near to those found at $295 \mathrm{~K}$ in Ref. 3 and to the pure dipole contributions. These agreements, together with the near coincidence of the individual off-diagonal components of the shift and dipole tensors in Ref. 3 , strongly suggest that the whole shift tensor is indeed temperature (and field) independent, and that the anisotropic THI is at least an order of mag- nitude smaller than the dipole interaction and the isotropic THI.

According to the spectroscopic work of Christensen, ${ }^{9}$ small structural changes occur in $\mathrm{LiTbF}_{4}$ between 1.3 and $300 \mathrm{~K}$, while this is not seen in $\mathrm{LiHoF}_{4 \cdot}{ }^{10}$ These changes in $\mathrm{LiTbF}_{4}$ influence the NMR line shifts both via the crystal-field-dependent susceptibility and through alterations in the shift tensors. Only the latter mechanism would show up in the results, since we use the measured susceptibility in the data treatment. As the results for $\mathrm{LiTbF}_{4}$ as well as for $\mathrm{LiHoF}_{4}$ lead to temperature-independent shift tensors, the changes observed by Christensen for $\mathrm{LiTbF}_{4}$ are consequently too small to influence the shift tensor significantly.

\section{CONCLUSION}

In the lithium rare-earth tetrafluorides, the isotropic THI between the rare-earth ionic moment and the $F$ nucleus is temperature independent from liquid-He temperature to room temperature, and field independent up to $40 \mathrm{kG}$. We have found the same temperature independence to be valid in rare-earth trifluorides, but in the narrower temperature region $77-300 \mathrm{~K}$.

The fluoride isotropic THI is found to be negative, and the absolute value to decrease on going to heavier rare earths. The anisotropic THI is. much smaller. These features are also in qualitative agreement with our findings for the trifluorides. The isotropic as well as the anisotropic THI between the rare-earth ionic moment and the Li nuclei is negligibly small.

\section{ACKNOWLEDGMENTS}

The authors are very grateful to Professor V. Frank for his continued interest during the experimental work and for his numerous suggestions towards improving and clarifying the manuscript. We also owe thanks to Dr. J. O. Rahbek for his construction of the high-frequency NMR oscillator. This research was supported in part by the Danish Natural Science Research Council under Grant No. 511-3575.
*Present address: HG-ER, L21, V9, Danfoss, DK-6430 Nordborg, Als, Denmark.

${ }^{1}$ A. Reuveni and B. R. McGarvey, J. Magn. Reson. 29, 21 (1978).

${ }^{2}$ P. E. Hansen, R. Nevald, and H. G. Guggenheim, Phys. Rev. B 17, 2866 (1978).

${ }^{3}$ P. E. Hansen and R. Nevald, Phys. Rev. B 16, 146 (1977).

${ }^{4}$ L. M. Holmes, T. Johansson, and H. J. Guggenheim,
Solid State Commun. 12, 993 (1973).

${ }^{5}$ P. E. Hansen, T. Johansson, and R. Nevald, Phys. Rev. B 12, 5315 (1975).

${ }^{6}$ I. Laursen and L. M. Holmes, J. Phys. C 7, 3765 (1974).

${ }^{7} \mathrm{~J}$. O. Rahbek (unpublished).

${ }^{8}$ R. Nevald and P. E. Hansen, Rev. Sci. Instrum. $\underline{49}$, 64 (1978).

${ }^{9}$ H. P. Christensen, Phys. Rev. B 17, 4060 (1978).

${ }^{10}$ H. P. Christensen (private communication). 